\title{
Spot-Light: Multimodal Projection Mapping on Food
}

\author{
Yui Kita $^{1}$ and Jun Rekimoto ${ }^{1,2}$ \\ ${ }^{1}$ Interfaculty Initiative in Information Studies, The University of Tokyo, \\ 7-3-1 Hongo, Bunkyo-ku Tokyo, 113-0033 Japan \\ ${ }^{2}$ Sony Computer Science Laboratories, Inc. 3-14-13 Higashigotanda, \\ Shinagawa-ku Tokyo, 141-0022 Japan \\ yuikita21@gmail.com, rekimoto@acm.org
}

\begin{abstract}
Spot-Light is a projection mapping system for foods that enriches dishes by projecting images or sounds on foods. Tastes of foods are constructed not only with simple gustation, but also with appearances and sounds. For better appearances, ordinary cooking methods such as food coloring agents or crunching sounds of pie contribute to modify these food properties. However, it is not possible to purely modify them because all ingredients are inevitable from chemical interruptions into the original food. Our system enhances these properties by visual and sound projection with a RGB camera, a depth camera, a parametric speaker and a projector.
\end{abstract}

Keywords: Food, Projection Mapping, Kitchen.

\section{Introduction}

\subsection{Cross-Modality on Dinning}

Appearances and sounds are important on a dinning experience as well as gustation. For example, some soft drinks provide various flavors only by visual difference although they contains mostly same ingredients. Professional chefs often use extra colors in recipes to modify the food appearances. For example, a recipe uses paprika's red as an additional color when using apple mint: the red color reminds guests the apple flavor which complements weak aroma of the apple mint. Thus, our resulting gustation is effected by prior perceptions (the effect is widely studied as crossmodality [1]). This suggests that modifications on appearances and sounds are effective to enrich the original dish.

\section{Spot-Light}

Spot-Light is a system that enhances such modalities. The system is in the shape of a desk light. For multimodal projections, a RGB camera, a depth camera, a parametric speaker and a projector are attached on the head part of the system. There are three functions in this system. The first function is to emphasize the surface color of the dish (for example, the saturation of green salad or browning of steaks). It uses a RGB 
camera and a depth camera for a precise projection mapping. The second function is to emphasize the sounds of the dish (for example, the crisply sound of baked pies or sounds of breaking a caramel of crème brûlée) so that it reminds guests extra rich textures of food. The third function is to project additional animations (for example, melting butters or surface shines).

We describe details of these functions and potential functions below.

\subsection{Appearance Augmentation for Food Coloring}

Chefs create better perceptions for foods by designing the food surface. As we mentioned above, the designing of the appearance is one of the designing process of gustation because the visual perception largely effects to the gustatory sensation.

Coloring is the basic of visual designing of food. The ordinary method to modify the color is to use natural or artificial colorings. However, there are some health risks when using these colorings. In addition, there are also limitations on their effects because they contain chemical materials that unexpectedly affect the original taste of food itself.

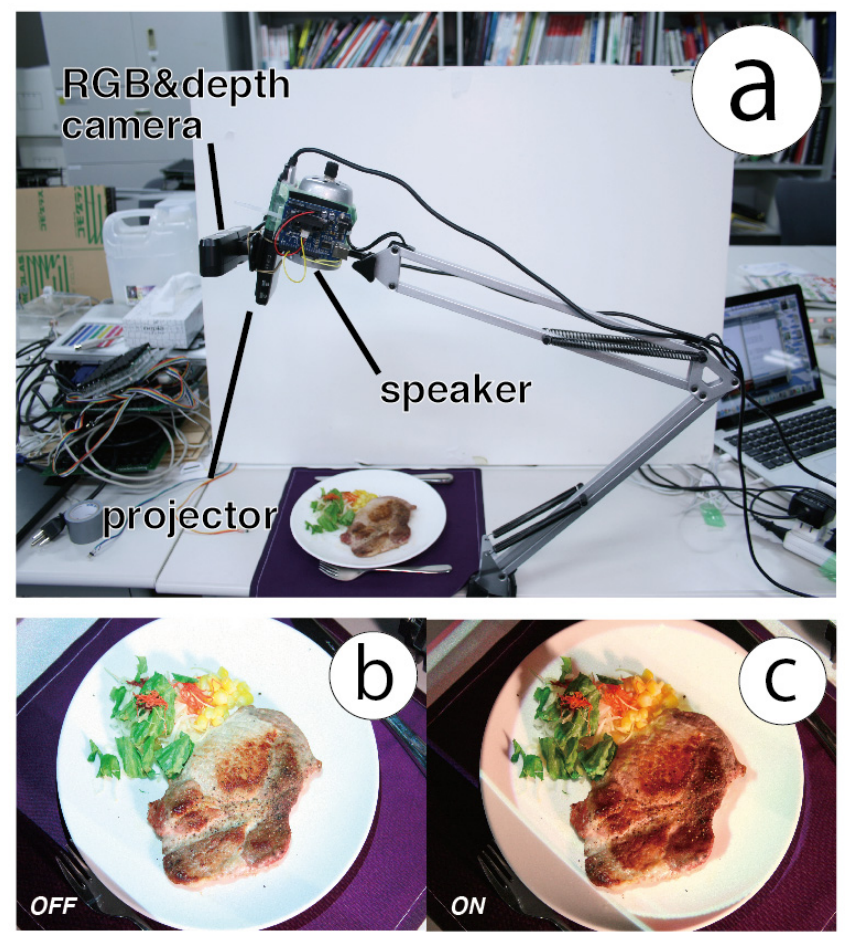

Fig. 1. Spot-Light consists of devices for multimodal projection mappings on the head part of a desk light (a). b and c shows the comparison of food's appearance without appearance augmentations (b) and with augmentations (c). The system first capture the RGB and depth image of the dish, and projects images that was processed so that it maximize the parts that effects positive gustation (for example, the saturation of green salad or browning of steaks). 
Spot-Light captures a RGB image of the dish, and projects modified images on it after processing the image so that it maximize the food's appearances. This function enables us to coloring the food without taking the heal risk, or changes of the original tastes. In addition, the system creates precise projections by using the depth camera in addition to the RGB camera: the depth information helps to provide warp-free projections. The precise projection mapping minimizes the artificial impression that results in a negative impression of food.

\subsection{Sound Augmentation for Food Texture}

Sounds also effect the gustation. Charles Spence [2] found that participants feel potato chips crisper when processed noise was presented while eating. Chewing jockey [3] is another project that enriches eating by giving additional chewing sounds while eating.

Spot-Light creates sounds of food such as breaking sounds of baked crispy pie or caramel of crème brûlée. To realize natural sound effects, we used a parametric speaker. The speaker can create sounds from a particular location of a surface with ultrasonic waves.

Our first prototype senses cutting gestures with a electrode and add extra crispy sounds to the precise location on the surface. The sound projection is programmed so that it makes sounds that are unique for each location.

\subsection{Animations for Impressive Food}

In addition to the appearance augmentation, animation projections are also provided. While the static image projection is effective to enhance the basis of the appearance, animations are more impressive. The impressiveness helps the guests to relate their memory to gustation and contributes intensive sensation. The system generates animations such as melting butter, up going smoke or surface shines that remind the guests a richer experience of dinning.

\section{Related Work}

DinningPresenter [4] projects images on a dining table. It contains a camera and a projector. The camera captures the image of the dinning table and projects image on it. The focus of this project is to enrich the whole experience of dinning. It projects colors and images to promote healthier dinning for kids, for instance.

Meta-Cookie is an AR system that leverages the cross-modality to enhance foods. It modifies the gustation or satisfaction by providing aromas or modified shape of cookie with an AR environment. Although Meta-Cookie is effective way to modify our perceptions, it is not practical because of large devices required for the AR environment. 


\section{Conclusion}

In this paper, we propose Spot-Light which enriches dishes by visual and sound projection mapping. This system is in the desk light shape with devices attached on the head part of it. The device includes a RGB camera, a depth camera, a parametric a speaker and a projector.

The system contains three functions. The first function is to capture the surface of food such as the brown area of steaks and projects extra visuals after processing the captured image so that it enriches the appearance. This function is supported by depth image captured by the depth camera, which helps a precise projection mapping that is important to eliminate the negative artificial impressive often occurs in simple projection mappings.

The second function is augmentations for the sound of food. The system adds sounds such as crispy sounds for baked pie when a knife touched on it. A parametric speaker is used to create sound on the surface.

The third function is animation projection. Animations such as melting butter on steaks are projected. The animation helps the guests to imagine gustation and helps intensive sensations.

By utilizing RGB and depth image, the system can project various properties on foods. For example, Spot-Light can be a real-time baking machine by attaching a laser.

Fukuchi [6] et al. used a laser for the use of baking the surface of food. They first captured the surface of the food and baked intended parts by processing the captured image. This suggests that Spot-Light can realize a real-time baking function with a laser.

\section{References}

1. Simner, J., Cuskley, C., Kirby, S.: What sound does that taste? Cross-modal mappings across gustation and audition. Perception 39(4), 553 (2010)

2. Zampini, M., Spence, C.: The role of auditory cues in modulating the perceived crispness and staleness of potato chips. Journal of Sensory Science 19, 347-363 (2004)

3. Koizumi, N., Tanaka, H., Uema, Y., Inami, M.: Chewing jockey: augmented food texture by using sound based on the cross-modal effect. In: Proceedings of the 8th International Conference on Advances in Computer Entertainment Technology, ACE 2011 (2011)

4. Mori, M., et al.: Dining Presenter: Augmented Reality system for a dining tabletop. In: Ajunct Proceedings of Ubicomp, pp. 168-169 (2009)

5. Narumi, T.: Meta cookie. In: ACM SIGGRAPH 2010 Posters. ACM (2010)

6. Fukuchi, K., et al.: Laser cooking: a novel culinary technique for dry heating using a laser cutter and vision technology. In: Proceedings of the ACM Multimedia 2012 Workshop on Multimedia for Cooking and Eating Activities. ACM (2012) 